\title{
EXTRAÇÃO DE COMPOSTOS BIOATIVOS DE SEMENTES DE MARACUJÁ-AZEDO (Passiflora edulis) UTILIZANDO LÍQUIDOS PRESSURIZADOS
}

\author{
F. M. BARRALES ${ }^{1}$, J. VIGANÓ $^{1}$, R. G. CORREA ${ }^{1}$ e J. MARTÍNEZ ${ }^{1}$ \\ ${ }^{1}$ Universidade Estadual de Campinas, Faculdade de Engenharia de Alimentos, \\ Departamento de Engenharia de Alimentos \\ E-mail para contato: manubarrales@gmail.com
}

\begin{abstract}
RESUMO - O objetivo deste trabalho foi extrair compostos fenólicos de sementes de maracujá e verificar a capacidade antioxidante dos extratos. Técnicas de extração utilizando líquidos pressurizados, como água e etanol, são consideradas ambientalmente corretas, pois estes solventes não oferecem riscos ambientais e ao produto. Os experimentos de extração seguiram um planejamento experimental composto central com os fatores temperatura $\left(30-80{ }^{\circ} \mathrm{C}\right)$ e percentual de etanol em água (0-100\%). As variáveis vazão de solvente, pressão e tempo de extração foram mantidas constantes. Após a extração, os extratos foram armazenados a $-18{ }^{\circ} \mathrm{C}$ e analisados quanto ao rendimento, fenólicos totais e capacidade antioxidante. As análises de fenólicos totais foram realizadas segundo o método FolinCiocalteu e a capacidade antioxidante por ensaios DPPH. Os resultados indicaram que maiores temperaturas e concentrações de etanol em água favorecem a obtenção de maiores percentuais de rendimento, fenólicos totais e capacidade antioxidante. A melhor condição obtida por este trabalho foi $55{ }^{\circ} \mathrm{C}$ e $100 \%$ etanol, em que se obteve $6,285 \%$ de rendimento, fenólicos totais igual a 322,166 mgAGE/100g e atividade antioxidante de $317,261 \mathrm{mgTE} / 100 \mathrm{~g}$.
\end{abstract}

\section{INTRODUÇÃO}

A produção, o comércio e o consumo de frutos tropicais exóticos têm aumentado nos últimos anos devido às suas propriedades sensoriais atrativas e ao reconhecimento de seu valor nutricional e terapêutico. Em muitos casos estes frutos não são somente consumidos na forma in natura. O maracujá-azedo (Passiflora edulis), utilizado pela indústria de alimentos em grande quantidade na fabricação de suco e polpa, é um exemplo.

Os processos de separação da polpa de maracujá produzem volumes consideráveis de resíduos devido à separação da casca, fibras da polpa e sementes. Estima-se que este material corresponde entre 60 e $70 \%$ da massa do fruto (Zeraik et al., 2010; Gerola et al., 2013). O desenvolvimento de técnicas que visam ao uso eficiente dos subprodutos agroindustriais pode agregar valor a estes materiais e reduzir impactos ambientais resultantes de seu descarte.

A valorização dos subprodutos agroindustriais está recebendo especial atenção nos últimos anos. Algumas pesquisas têm avaliado a conversão de subprodutos em ingredientes para alimentos e componentes para fármacos (Santana-Méridas et al., 
2012). Outras indicam que partes do fruto e da planta de espécies do gênero Passiflora são boas fontes de polifenois (Oliveira et al., 2009; Martinez et al., 2012), ácidos graxos (Liu et al., 2009) e carotenoides (Ferreira et al., 2013), compostos de alto valor nutricional, aos quais se atribui diversos benefícios à saúde. A obtenção destes compostos pode originar uma gama de produtos comerciais, seja matéria-prima para processos secundários, substitutos para ingredientes ou ingredientes de novos produtos (Martinez et al., 2012). Entretanto, para explorar estes recursos, estratégias comercialmente viáveis para a extração e disponibilização desses compostos precisam ser desenvolvidas.

A extração com líquidos pressurizados (Pressurized liquid extraction - PLE) tem recebido especial atenção nos últimos anos, pois permite a extração rápida, com menor consumo de solventes e alta seletividade dos solutos (Herrero et al., 2004). Além disso, há possibilidade de operar com solventes como água e etanol, o que torna a técnica ambientalmente correta e economicamente viável em relação aos métodos convencionais de extração, em que grandes quantidades de solventes, longos tempos de extração e altas temperaturas são requeridas (Ju e Howard, 2003; Santos et al., 2013).

Com base no exposto, o objetivo deste trabalho foi realizar extrações de sementes de maracujá-azedo utilizando fluidos pressurizados e avaliar os extratos quanto a conteúdo de fenólicos totais e capacidade antioxidante in vitro.

\section{MATERIAL E MÉTODOS}

\subsection{Pré-tratamento da matéria-prima}

Maracujás da espécie Passiflora edulis foram adquiridos no comércio local de Campinas-SP. As sementes foram separadas da polpa com o auxilio de liquidificador e peneira, cuidando para separá-las completamente da fibra da polpa, sem rompê-las. Posteriormente, foram levadas à estufa com circulação de ar a $60{ }^{\circ} \mathrm{C}$, onde permaneceram por 24 horas. Após o processo de secagem, as sementes apresentaram teor de umidade igual a $0,025 \pm 0,001 \mathrm{~kg}$ água $/ \mathrm{kg}_{\text {amostra. }}$ Para finalizar o pré-tratamento, as sementes foram trituradas.

\subsection{Extração com líquido pressurizado (PLE)}

O trabalho foi desenvolvido no Laboratório de Alta Pressão em Engenharia de Alimentos (LAPEA-DEA-FEA-UNICAMP). As extrações foram realizadas no equipamento representado no fluxograma da Figura 1. Diferentes composições de solvente foram bombeadas a partir do reservatório até a célula de extração. Para cada ensaio, um leito de partículas foi empacotado, utilizando-se aproximadamente 5 gramas de sementes e completando o restante do volume da célula $(100 \mathrm{~mL})$ com esferas de vidro. A célula de extração possui uma camisa acoplada a um banho termostático para controle da temperatura. A pressão foi controlada pelo ajuste da válvula micrométrica. Os extratos foram coletados em frascos com proteção da luz no final da linha. Ao término da extração, os extratos foram pesados. Após a determinação de rendimento global, os extratos foram rota-evaporados durante uma hora a $38{ }^{\circ} \mathrm{C}$, liofilizados e armazenados a $-24^{\circ} \mathrm{C}$. 


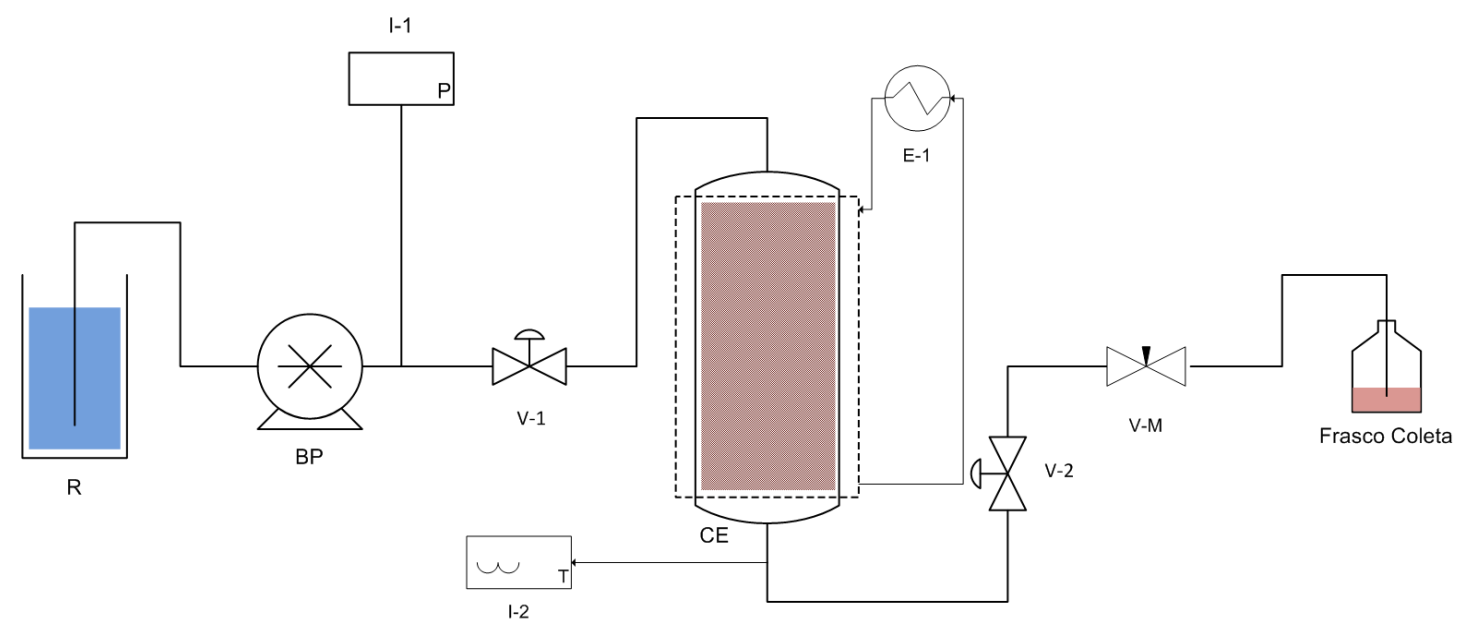

Figura 1 - Fluxograma da unidade de extração. R: reservatório de solvente; BP: bomba HPLC; I-1: indicador de pressão; CE: célula de extração; V-1 e V-2: válvulas de bloqueio; E-1: banho termostático; I-2: indicador de temperatura; V-M: válvula micrométrica.

Os experimentos de extração seguiram um planejamento experimental composto central (Tabela 1), cujas variáveis foram temperatura e percentual de etanol em água. Os parâmetros $x_{1}$ e $x_{2}$ representam as variáveis codificadas (temperatura e percentual de etanol respectivamente). A pressão, o tempo de extração e a vazão de solvente foram mantidos constantes, $10 \mathrm{MPa}, 60 \mathrm{~min}$. e $3 \mathrm{~mL} / \mathrm{min}$., respectivamente. A ordem de realização dos experimentos foi aleatória.

Tabela 1 - Planejamento experimental composto central

\begin{tabular}{|c|c|c|c|c|}
\hline Ensaio & $\begin{array}{c}\text { Temperatura } \\
\left({ }^{\circ} \mathrm{C}\right)\end{array}$ & $\begin{array}{c}\text { Etanol } \\
(\%)\end{array}$ & $x_{1}$ & $x_{2}$ \\
\hline 1 & 37 & 15 & -1 & -1 \\
\hline 2 & 73 & 15 & +1 & -1 \\
\hline 3 & 37 & 85 & -1 & +1 \\
\hline 4 & 73 & 85 & +1 & +1 \\
\hline 5 & 30 & 50 & $-1,42$ & 0 \\
\hline 6 & 80 & 50 & $+1,42$ & 0 \\
\hline 7 & 55 & 0 & 0 & $-1,42$ \\
\hline 8 & 55 & 100 & 0 & $+1,42$ \\
\hline 9 & 55 & 50 & 0 & 0 \\
\hline 10 & 55 & 50 & 0 & 0 \\
\hline 11 & 55 & 50 & 0 & 0 \\
\hline
\end{tabular}

\subsection{Rendimento global}

Uma alíquota de massa conhecida de cada extrato foi submetida à análise de sólidos secos em estufa a vácuo a $60{ }^{\circ} \mathrm{C}$, segundo metodologia da AOAC (1997). O percentual de sólidos secos foi relacionado com a massa de extrato para obter a massa total de soluto recuperado. Com essa informação foi calculado o rendimento global da extração, como mostra a Equação 1.

$X_{0}(\%)=100 \times \frac{m_{\text {soluto }}}{\mathrm{F}}$ 
Onde: $X_{0}$ é o rendimento global; $m_{\text {soluto }}$ é a massa total de soluto recuperado; e $F$ é a massa de amostra usada para empacotar o leito.

\subsection{Análise de fenólicos totais (FT)}

A determinação de teor de compostos fenólicos foi realizada pelo método de Folin-Ciocalteu, descrito por Singleton et al. (1999). A partir do extrato seco, prepararam-se diluições aquosas dos extratos em duas concentrações 0,4 e $0,2 \mathrm{mg} / \mathrm{mL}$. Alíquotas de $0,5 \mathrm{~mL}$ de amostra dissolvida ou água destilada (branco de calibração) foram misturadas com $0,5 \mathrm{~mL}$ de reagente Folin-Ciocalteu, efeituando-se agitação manual por $15 \mathrm{~s}$. Após 3 minutos, adicionou-se $0,5 \mathrm{~mL}$ de solução saturada de carbonato de sódio e a solução foi diluída com água destilada para completar $5 \mathrm{~mL}$. A mistura foi mantida no escuro por 150 minutos para reagir, logo a absorbância foi medida a $760 \mathrm{~nm}$ em espectrofotômetro UV-vis. A concentração de compostos fenólicos foi estimada usando uma curva de calibração realizada com ácido gálico (15, 20, 30, 40, 50, 60 e $70 \mu \mathrm{g} / \mathrm{mL})$.

\subsection{Análise da capacidade antioxidante}

A determinação de capacidade antioxidante in vitro foi realizada segundo a metodologia de DPPH (2,2-diphenyl-1-picryl-hidrazil) descrita por Rufino et al. (2007), com modificações. Prepararam-se diluições do extrato seco em etanol, nas concentrações de 1 e $0,5 \mathrm{mg} / \mathrm{mL}$. Posteriormente foi preparada uma solução de reagente DPPH $(60 \mu \mathrm{M})$, dissolvendo 4,8 mg de reagente DPPH em $200 \mathrm{~mL}$ de etanol, logo uma solução estoque de Trolox $(2000 \mu \mathrm{M})$ foi preparada, dissolvendo $25 \mathrm{mg}$ de reagente Trolox em $50 \mathrm{~mL}$ de etanol, a partir da solução estoque foram preparadas em balões volumétricos de $5 \mathrm{~mL}$ soluções com as seguintes concentrações: 50, 100, 200, 400, 600, 800, 1000 e $1200 \mu \mathrm{M}$; para realizar a curva de calibração de Trolox. Em ambiente escuro, transferiu-se para tubos de ensaio alíquotas de $0,1 \mathrm{~mL}$ de solução de Trolox, etanol (controle) ou diluição de amostra e logo foi adicionado 3,9 mL de solução de reagente DPPH, após agitação manual por $30 \mathrm{~s}$ os tubos foram mantidos no escuro a temperatura ambiente durante 40 minutos, para que a reação ocorresse. Transcorrido esse tempo se realizou a leitura da absorbância no comprimento de onda de $515 \mathrm{~nm}$ em espectrofotômetro UV-vis, usando o etanol como branco.

\section{RESULTADOS E DISCUSSÃO}

Os resultados do rendimento global de cada experimento de extração são apresentados na Tabela 2. Pode-se observar que os maiores rendimentos foram obtidos para os níveis altos de porcentagem de etanol com fraca influência da temperatura. $O$ diagrama de Pareto apresentado na Figura 2 (A) mostra que somente a porcentagem de etanol (linear e quadrática) teve efeito significativo a $95 \%$ de confiança. O maior rendimento $(6,285 \%)$ foi obtido a $55{ }^{\circ} \mathrm{C}$ e $100 \%$ de etanol. Isto mostra que para o rendimento global, etanol como solvente possui melhor desempenho que a água para recuperar extratos de sementes de maracujá. Liu et al. (2009) obtiveram óleo de sementes de maracujá através de extração supercrítica com $\mathrm{CO}_{2}$. $\mathrm{O}$ rendimento atingido por esses autores $(25,98 \%)$ foi substancialmente superior ao obtido por esse trabalho. Isso se deve ao fato do $\mathrm{CO}_{2}$ ter melhor afinidade com óleo do que o etanol. No entanto, dependendo do objetivo, o rendimento global pode não medir a eficiência do processo. No caso deste trabalho, cujo objetivo é a extração de compostos bioativos, é mais interessante a obtenção de extratos com alto teor de compostos fenólicos e elevada 
capacidade antioxidante.

Tabela 2 - Rendimento global, fenólicos totais (FT) e atividade antioxidante (DPPH) dos extratos obtidos de sementes de maracujá-azedo - valores expressos pela média \pm desvio padrão

\begin{tabular}{|c|c|c|c|r|r|}
\hline Ensaio & $\mathrm{T}\left({ }^{\circ} \mathrm{C}\right)$ & $\%$ Etanol & $X_{0}(\%)$ & $\begin{array}{c}\text { FT } \\
(\mathrm{mgAGE} / 100 \mathrm{~g})\end{array}$ & DPPH (mgTE/100g) \\
\hline 1 & 37 & 15 & $0,939 \pm 0,022$ & $41,456 \pm 0,5$ & $28,704 \pm 2,3$ \\
\hline 2 & 73 & 15 & $0,956 \pm 0,006$ & $68,195 \pm 0,8$ & $12,870 \pm 0,7$ \\
\hline 3 & 37 & 85 & $1,096 \pm 0,033$ & $92,941 \pm 0,1$ & $117,672 \pm 9,1$ \\
\hline 4 & 73 & 85 & $1,923 \pm 0,007$ & $255,885 \pm 0,3$ & $214,959 \pm 3,9$ \\
\hline 5 & 55 & 50 & $1,016 \pm 0,002$ & $149,773 \pm 0,9$ & $115,481 \pm 2,5$ \\
\hline 6 & 55 & 50 & $0,791 \pm 0,003$ & $120,327 \pm 3,6$ & $97,706 \pm 2,8$ \\
\hline 7 & 55 & 50 & $1,040 \pm 0,006$ & $170,422 \pm 3,1$ & $118,652 \pm 7,4$ \\
\hline 8 & 55 & 100 & $6,285 \pm 0,001$ & $322,166 \pm 2,8$ & $317,261 \pm 7,6$ \\
\hline 9 & 80 & 50 & $1,239 \pm 0,006$ & $286,658 \pm 0,7$ & $194,461 \pm 13,6$ \\
\hline 10 & 55 & 0 & $0,696 \pm 0,001$ & $21,808 \pm 0,1$ & $38,478 \pm 0,95$ \\
\hline 11 & 30 & 50 & $0,788 \pm 0,027$ & $94,013 \pm 0,9$ & $67,654 \pm 0,45$ \\
\hline
\end{tabular}

*AGE: Ácido gálico equivalente

**TE: Trolox equivalente

Na Tabela 2 são apresentados também os resultados das análises de fenólicos totais e atividade antioxidante obtida pelo método DPPH. Pode-se observar que os maiores valores de fenólicos totais e capacidade antioxidante foram obtidos com o aumento da porcentagem de etanol e temperatura. Para fenólicos totais somente os fatores porcentagem de etanol e temperatura (linear) tiveram efeitos significativos a 95\% de confiança. Para DPPH, porcentagem de etanol (linear), temperatura (linear) e interação entre ambos tiveram efeitos significativos a 95\% de confiança (Figura 2 (A) e (B)).

O Ensaio 8 mostrou melhor desempenho nas avaliações de compostos fenólicos totais e capacidade antioxidante, 322,166 mgAGE/100g e 317,261 mgTE/100g, respectivamente. Outras pesquisas realizaram estas mesmas determinações para resíduos e polpa de maracujá. Martinez et al. (2012) trabalharam com resíduos do maracujá (casca, sementes e restos de polpa, secos e triturados) e os submeteram à extração com dois solventes (metanol/acetona e etanol) a pressão e temperatura ambiente por $24 \mathrm{~h}$. O resultado de compostos fenólicos totais obtido por esses autores na extração com metanol/acetona é próximo ao deste trabalho (246 mgAGE/100g). Por outro lado, a atividade antioxidante foi inferior $(127,5 \mathrm{mgTE} / 100 \mathrm{~g})$. Oliveira et al. (2009) trabalharam com o mesmo tipo de resíduos de Martinez et al. (2012) e os submeteram à extração em Soxhlet por $4 \mathrm{~h}$ com $n$-hexano e posteriormente por mais $4 \mathrm{~h}$ com metanol, sempre a $60{ }^{\circ} \mathrm{C}$ e pressão atmosférica. Os extratos obtidos por metanol apresentaram conteúdo de compostos fenólicos totais igual a $103 \mathrm{mgAGE} / 100 \mathrm{~g}$ e capacidade antioxidante não foi reportada em termos de $\mathrm{mgTE} / 100 \mathrm{~g}$. Os extratos obtidos por $n$-hexano não exibiram capacidade antioxidante e consequentemente não foram analisados quanto a compostos fenólicos. Valores próximos ao alcançados neste trabalho foram obtidos por Souza et al. (2012) quando da extração de polpa de maracujá doce. Estes autores reportaram teor de compostos fenólicos de 245,36 mgAGE/100g e atividade antioxidante de $271 \mathrm{mgTE} / 100 \mathrm{~g}$. Com base nestes resultados pode-se inferir 
que o extrato das sementes de maracujá, obtido por fluidos pressurizados, pode ser uma fonte de igual ou maior quantidade de compostos bioativos do que a polpa.

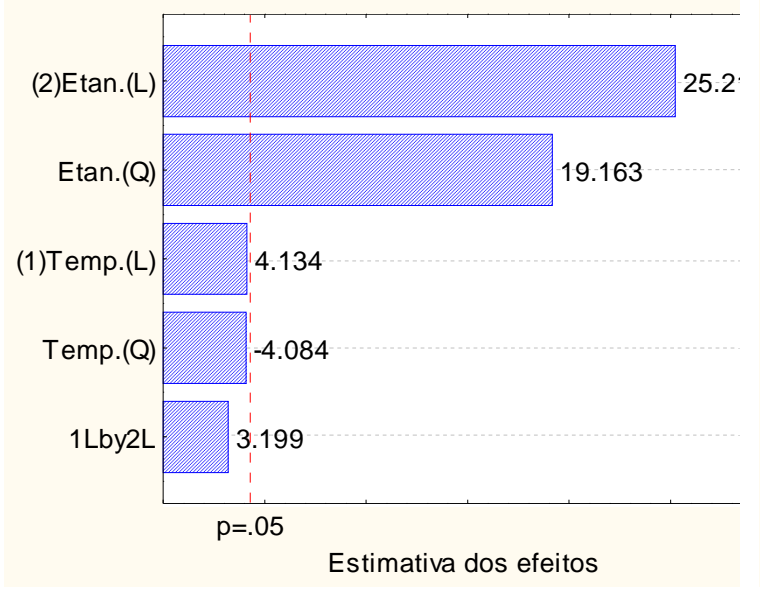

(A)

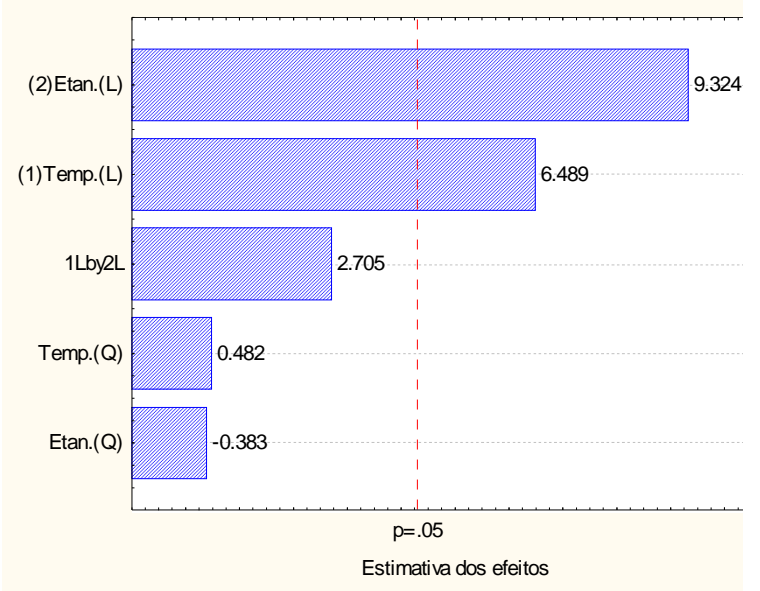

(B)

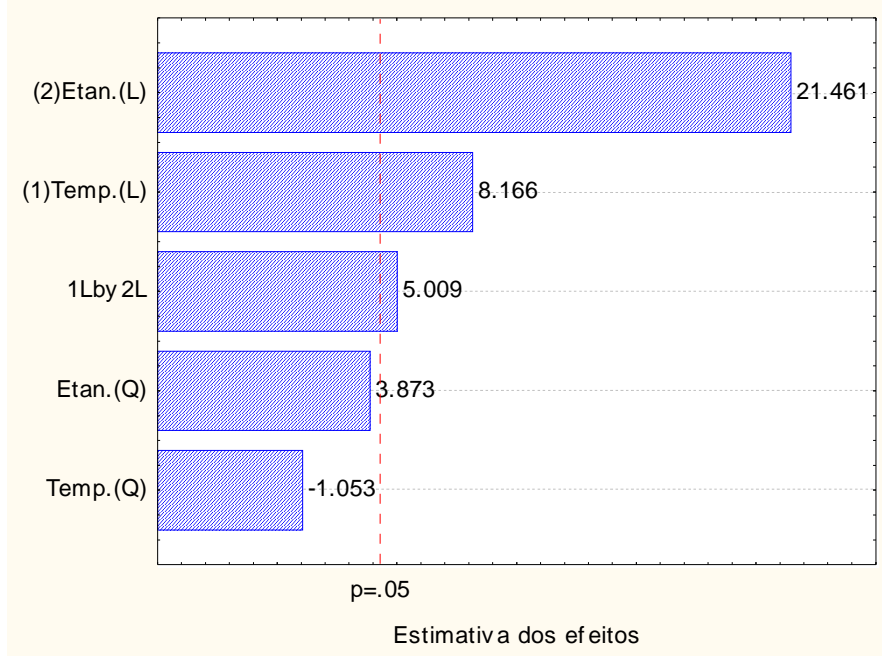

(C)

Figura 2 - Diagrama de Pareto para estimativa dos efeitos, Rendimento (A), Fenólicos totais (B) e DPPH (C)

\section{CONCLUSÕES}

A técnica de extração com fluidos pressurizados demonstrou ser apropriada para a extração de compostos bioativos em sementes de maracujá. O ensaio com maior rendimento global coincidiu com os melhores resultados de compostos fenólicos totais e capacidade antioxidante, ou seja, $55{ }^{\circ} \mathrm{C}$ e $100 \%$ de etanol, em que se obteve $6,285 \%$, $322,166 \mathrm{mgAGE} / 100 \mathrm{~g}$ e $317,261 \mathrm{mgTE} / 100 \mathrm{~g}$. A melhor condição deste trabalho resultou em valores comparáveis com os resultados disponíveis na bibliografia para a fruta inteira e somente polpa.

\section{REFERÊNCIAS}

AOAC. Official methods of analysis of the Association of Official Analytical Chemistry. 16th ed. Gaithersburg, USA: AOAC International, 1997. 
FERREIRA, B. S.; DE ALMEIDA, C. G.; LE HYARIC, M.; DE OLIVEIRA, V. E.; EDWARDS, H. G. M.; DE OLIVEIRA, L. F. C. Raman Spectroscopic Investigation of Carotenoids in Oils from Amazonian Products. Spectrosc. Lett., v. 46, n. 2, p. 122-127, 2013.

GEROLA, G. P.; BOAS, N. V.; CAETANO, J.; TARLEY, C. R. T.; GONÇALVES, A. C.; DRAGUNSKI, D. C. Utilization of passion fruit skin by-product as lead (II) ion biosorbent. Water, Air, Soil Poll., v. 224, n. 2, 2013.

HERRERO, M.; IBÁÑEZ, E.; SEÑORÁNS, J.; CIFUENTES, A. Pressurized liquid extracts from Spirulina platensis microalga. J. Chromatogr., v. 1047, n. 2, p. 195203, 2004.

JU, Z. Y.; HOWARD, L. R. Effects of solvent and temperature on pressurized liquid extraction of anthocyanins and total phenolics from dried red grape skin. J. Agric. Food Chem., v. 51, p. 5207-5213, 2003.

LIU, S.; YANG, F.; ZHANG, C.; JI, H.; HONG, P.; DENG, C. Optimization of process parameters for supercritical carbon dioxide extraction of Passiflora seed oil by response surface methodology. The J. of Supercritical Fluids, v. 48, n. 1, p. 9-14, 2009.

MARTINEZ, R.; TORRES, P.; MENESES, M. A.; FIGUEROA, J. G.; PÉREZÁLVAREZ, J. A.; VIUDA-MARTOS, M. Chemical, technological and in vitro antioxidant properties of mango, guava, pineapple and passion fruit dietary fibre concentrate. Food Chemistry, v. 135, n. 3, p. 1520-6, 2012.

OLIVEIRA, A. C.; VALENTIM, I. B.; SILVA, C. A.; BECHARA, E. J. H.; BARROS, M. P.; MANO, C. M.; GOULART, M. O. F. Total phenolic content and free radical scavenging activities of methanolic extract powders of tropical fruit residues. Food Chemistry, v. 115, n. 2, p. 469-475, 2009.

RUFINO, M. D. S. M.; ALVES, R. E.; DE BRITO, E. S.; DE MORAIS, S. M.; SAMPAIO, C. D. G.; PÉREZ-JIMÉNEZ, J.; SAURA-CALIXTO, F. Metodologia Científica Determinação da Atividade Antioxidante Total em Frutas pela Captura do Radical Livre. Comunicado Técnico (Embrapa Agroindústria Tropical. Online), p. 0-3, 2007.

SANTANA-MÉRIDAS, O; GONZÁLEZ-COLOMA, A.; SÁNCHEZ-VIOQUE, R. Agricultural residues as a source of bioactive natural products. Phytochemistry Reviews, v. 11, n. 4, p. 447-466, 2012.

SANTOS, D. T.; AlBARELli, J. Q.; BEPPU, M. M.; MEIRELES, M. A. A. Stabilization of anthocyanin extract from jabuticaba skins by encapsulation using supercritical $\mathrm{CO}_{2}$ as solvent. Food Res. Int., v. 50, n. 2, p. 617-624, 2013.

SINGLETON, V. L.; ORTHOFER, R.; LAMUELA-RAVENTOS, R. M. Analysis of total phenols and other oxidation substrates and antioxidants by means of FolinCiocalteu reagent. Method. Enzymol., v. 299, p. 152-178, 1999.

SOUZA, V. R.; PEREIRA, P. A. P.; QUEIROZ, F.; BORGES, S. V.; DE DEUS SOUZA CARNEIRO, J. Determination of bioactive compounds, antioxidant activity and chemical composition of Cerrado Brazilian fruits. Food Chemistry, v. 134, n. 1, p. 381-386, 2012.

ZERAIK, M. L.; PEREIRA, C. A. M.; ZUIN, V. G.; YARIWAKE, J. H. Maracujá: um alimento funcional? Braz. J. of Pharmacog., v. 20, n. 3, p. 459-471, 2010. 